\title{
Article \\ Protons Interaction with Nomex Target: Secondary Radiation from a Monte Carlo Simulation with Geant4
}

\author{
Filomena Loffredo ${ }^{1, * \mathbb{D}}$, Emanuele Vardaci ${ }^{2,3}$, Davide Bianco ${ }^{4} \mathbb{( D}$, Antonio Di Nitto ${ }^{2,3}$ and and aria Quarto ${ }^{1}(\mathbb{D})$ \\ 1 Advanced Biomedical Science Department, University of Naples "Federico II", Via S. Pansini, \\ 80131 Naples, Italy; maria.quarto@unina.it \\ 2 Department of Physics, "E. Pancini", University of Naples, Federico II, Corso Umberto I, 80138 Naples, Italy; \\ vardaci@na.infn.it (E.V.); antonio.dinitto@unina.it (A.D.N.) \\ 3 National Institute for Nuclear Physics (INFN), 80126 Naples, Italy \\ 4 Italian Aerospace Research Centre (CIRA), Via Maiorise, 81043 Capua, Italy; d.bianco@cira.it \\ * Correspondence: filomena.loffredo@unina.it
}

Citation: Loffredo, F.; Vardaci, E.; Bianco, D.; Di Nitto, A.; Quarto, M. Protons Interaction with Nomex Target: Secondary Radiation from a Monte Carlo Simulation with Geant4. Appl. Sci. 2022, 12, 2643. https:// doi.org/10.3390/app12052643

Academic Editor: Maria

Filomena Botelho

Received: 25 January 2022

Accepted: 2 March 2022

Published: 3 March 2022

Publisher's Note: MDPI stays neutral with regard to jurisdictional claims in published maps and institutional affiliations.

Copyright: (C) 2022 by the authors. Licensee MDPI, Basel, Switzerland. This article is an open access article distributed under the terms and conditions of the Creative Commons Attribution (CC BY) license (https:// creativecommons.org/licenses/by/ $4.0 /)$.

\begin{abstract}
The study of suitable materials to shield astronauts from Galactic Cosmic Rays (GCR) is a topic of fundamental importance. The choice of the material must take into account both the secondary radiation produced by the interaction between primary radiation and material and its shielding ability. The physics case presented here deals with the interaction of a proton beam with a Nomex shield, namely, a target material with a mass thickness of $20 \mathrm{~g} \mathrm{~cm}^{-2}$. The study was conducted with the simulation code DOSE based on the well-known simulation package Geant4. This article shows the properties of secondary radiations produced in the target by the interaction of a proton beam in an energy range characterizing the GCR spectrum. We observed the production of ions of masses and charges lower than the chemical elements that make up Nomex, and also a significant production of neutrons, protons, and $\alpha$ particles.
\end{abstract}

Keywords: Nomex target; MC simulation; Geant4; protons; secondary radiation

\section{Introduction}

Interplanetary travels are a real challenge for crews due to the very different living conditions from those in which humans usually live on Earth. Physical stress due to microgravity and the mixed composition of space radiation represent a potential showstopper to human exploration of space. In the space environment, there are energetic particles of different origins [1]. The dominant radiation with a very wide spectrum in energy, up to about $0.1 \mathrm{TeV}$, is constituted by the Galactic Cosmic Rays (GCR). The GCR [2] are composed of protons $(87 \%)$, alpha particles $(12 \%)$, and a relatively small amount of heavier nuclei $(\sim 1 \%)$ arriving from outside the heliosphere [3-8]. These particles continuously enter the solar cavity and are isotropically distributed. The energy spectrum of GCR is described by the differential intensity or differential flux $\varphi(E)$. The cyclical variations of the sun's magnetic fields coupled with the interactions of the GCR with the solar wind modulate the spectrum of the GCR [9]. A differential energy spectrum for hydrogen can be obtained by using the SPENVIS \{SPace ENVironment Information System\} software (version 4.6.10) [10], as shown in Figure 1.

The spectrum has been normalized to the maximum and ranges from 50 to $1000 \mathrm{MeV}$. The GCR CREME96 model [11] implemented in SPENVIS is based on the semiempirical model of Nymmik [12,13]. This model fixes the parameters that describe the variations of the flux of the particles (electrons, protons, and nuclei) that make up the GCR with the energy of $10 \div 10^{5} \mathrm{MeV} / \mathrm{A}$ in the space immediately surrounding the Earth beyond the terrestrial magnetosphere. 
Ion spectrum (GCR)

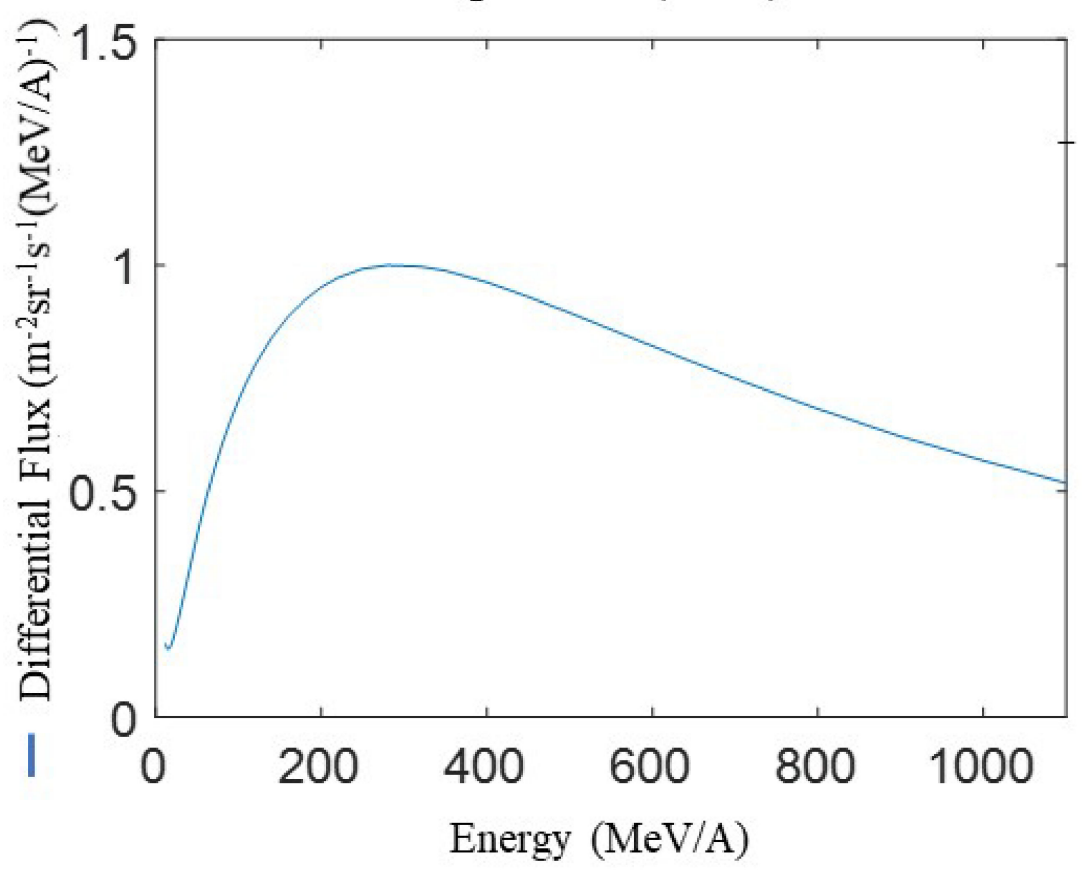

Figure 1. The differential energy spectra for hydrogen obtained using the SPENVIS software.

Currently, shielding is the only simple countermeasure to prevent exposure to radiation. Protection of astronauts from cosmic radiation, therefore, involves inevitable compromises in the design of equipment, for instance, suits and storm shelters, and concerns construction materials of the spacecraft. The high energy part of cosmic radiation and the consequent physical processes activated by the interaction between radiation and matter, such as nuclear reactions, makes the description of secondary radiation, potentially more harmful, very complex. It is, therefore, crucial to study (1) how cosmic radiation interacts with the shielding materials by selecting the possible physical processes involved, and (2) if the secondary radiation produced can be harmful, having the potential to escape from the shielding material.

This work shows the results obtained by a Monte Carlo simulation of the proton-shield interaction by taking into account the molecular constitution of the shield. For this specific work, we have used the Nomex material. The physical models of the electromagnetic and hadronic interactions were implemented in the code DOSE developed on the basis of the tool Geant4, as discussed in detail in previous work [14]. The code DOSE has been validated using tabulated and experimental data available only for $1 \mathrm{GeV}$ protons [14,15].

The aim of the present work is to extend the calculation of the production of secondary radiation in the target in a wider energy range characterizing the GCR spectrum in Figure 1. Calculations were performed at different energy values of the primary proton beam in order to explore the reliability of the code DOSE in describing the physical interaction processes, i.e., the nuclear reactions that take place and, hence, the consequential production of secondary particles.

The choice of the proper material for shielding also concerns its ability to minimize the escape of secondary radiation. In future work, we will show the implementation of the transport of secondary radiation with the aim to evaluate the dose absorbed by the astronauts behind the shield. The present work is to be considered as a natural continuation of the work reported in [15]. 


\section{Materials and Method}

Physical Processes

A short overview of the physical processes implemented in DOSE is given for completeness. A charged particle, such as a proton, moving in a material, loses energy almost continuously by inelastic Coulomb interaction with electrons and is slightly deflected from its initial direction. The interaction with the atomic nuclei gives rise either to elastic/inelastic collisions or nuclear reactions. In particular:

- Inelastic collisions with the atomic electrons of the material produce ionization or excitation of the atoms of the medium and energy loss of the charged incident particle.

- Elastic collisions in the Coulomb field of nuclei, although less frequent, lead to negligible energy loss while significantly changing the trajectory of the incident particle.

The amount of energy loss in each collision in both cases is a very small fraction of the initial kinetic energy of the incident particle. However, since the number of collisions per unit of travel is very high, the result is a significant cumulative energy loss with small fluctuations. The interaction of the charged particles is essentially of the electromagnetic type. Electromagnetic interactions are predominantly elastic collisions, i.e., collisions in which energy is transferred from one particle to another, but the oncoming particles retain their identity. However, there are numerous mechanisms of particle production in the interactions of high-energy particles in matter. The high energy protons (up to $0.1 \mathrm{TeV}$ ) that interact with a material give rise to nuclear collisions with the atomic nuclei in the shield and may produce hadrons (protons, neutrons, charged pions) or other nuclei, which in turn can have further nuclear collisions; other particles, such as neutral pions will decay almost instantly and whose products may initiate electromagnetic showers. In addition to fast hadrons, nuclear collisions also give rise to low-energy $(\mathrm{MeV})$ neutrons, protons, light ions (alpha particles), and gamma rays, which are emitted during the de-excitation of the target nuclei. Neutrons can be highly penetrating and can escape the material unless they undergo multiple elastic nuclear collisions until they thermalize and are captured. Eventually, the residual nuclei of elastic and inelastic collisions can also further interact with neighboring nuclei [16].

In this scenario, the type of secondary particles and their absorption inside the shield are critical properties of the shielding material. Secondary radiation escaping the shield might be less harmful than the primary one (preferred feature) or can be even more harmful, depending on the nature of the incident flux and the chemical composition of the shield. The effects of nuclear interactions on the radiation environment behind the shield are very different in the two cases: proton-nucleus collisions tend to make the environment behind the shield more dangerous [17-19], whereas nucleus-nucleus collisions tend to do the opposite [20].

\section{Geant4 Tool and DOSE Code}

Geant4 is a versatile and powerful toolkit to simulate the passage of particles through matter [21,22]. It includes a large variety of physics functionality for each particle type in the energy range from a few $\mathrm{eV}$ to several $\mathrm{TeV}$. It is possible to construct a detailed geometry that reproduces the irradiation conditions. Geant 4 allows users to choose among a wide range of models, driven by theory or data or based on parameterizations to describe the different physical phenomena depending on particle type and energy range. With respect to other general-purpose Monte Carlo systems for particle transport, Geant 4 is characterized by the peculiarity of encompassing a very large variety of physics modeling options [23]. Users are responsible for selecting the physics configuration of their experimental applications; the nature of Geant 4 as a toolkit prevents the definition of any "default" physics configuration.

The validation process of the code DOSE was carried out in two steps: (1) comparison of calculation results with experimental data available in the NIST database [14], i.e., Validation of the Electromagnetic Physical Processes; (2) comparison of simulation results with the experimental data collected at the NASA Space Radiation Laboratory in Brookhaven, namely, measurements performed by bombarding an aluminum slab with a $1 \mathrm{GeV}$ proton 
beam [14,24-26]. The flowchart in Figure 2 shows the processes implemented in DOSE. A detailed description of the models and the energy range of validity associated with each process are reported in the Physics Reference Manual [27].

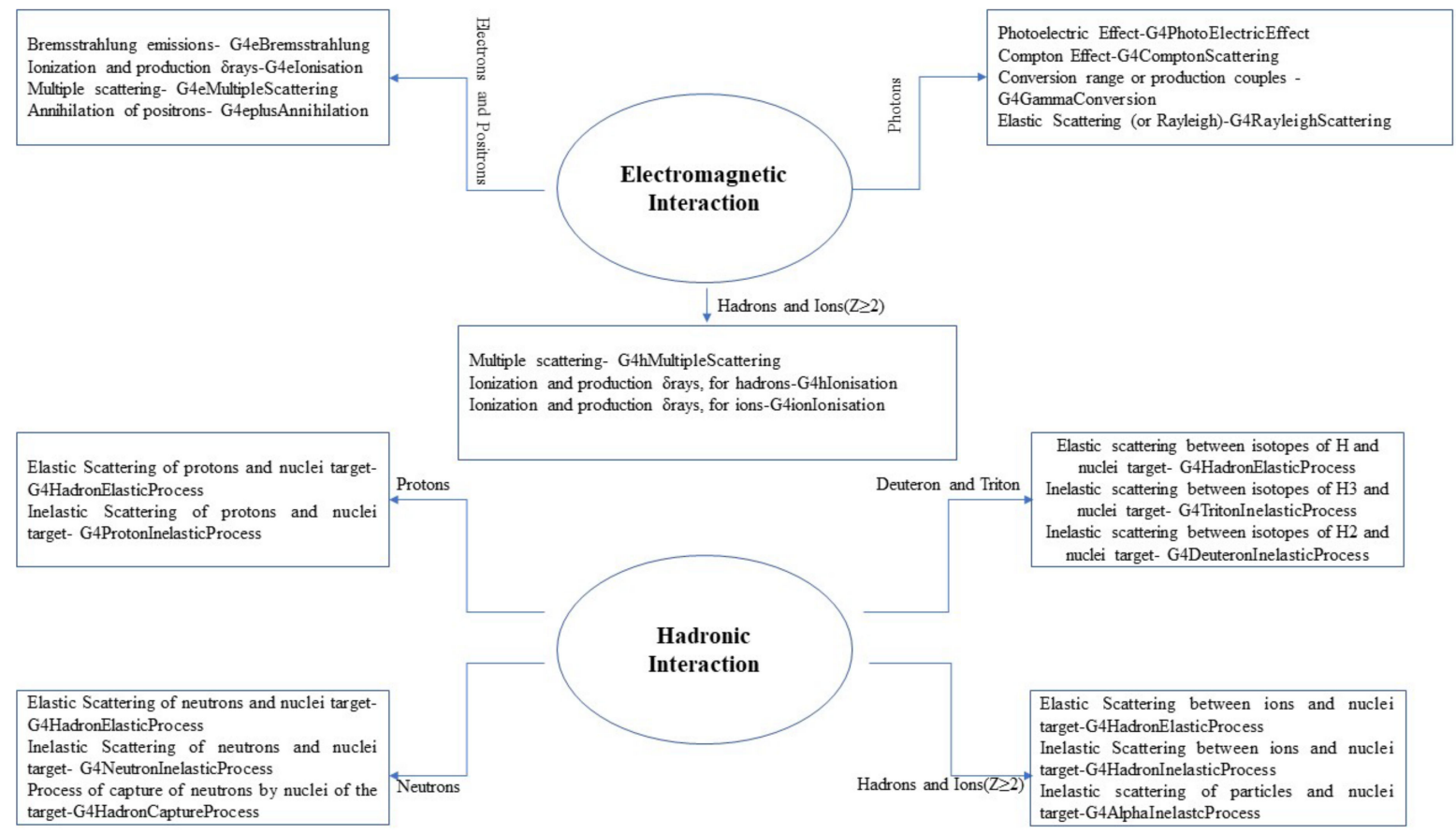

Figure 2. Flowchart concerning the processes implemented in the DOSE application.

\section{Geometry Used in the Calculation and Choice of the Shield Material}

In this work, the calculations are performed considering the experimental setup, which is shown schematically in Figure 3. The simulated geometry consists of an extended proton source, with a diameter of $20 \mathrm{~cm}$, energy ranges from $50 \mathrm{MeV}$ to $1000 \mathrm{MeV}$, in a vacuum. As a target, instead of the aluminum used in Loffredo et al., 2017 [14], we now use a Nomex slab $\left(\rho \sim 1.15 \mathrm{~g} / \mathrm{cm}^{3}\right)$, in the shape of a parallelepiped, with a thickness of $20 \mathrm{~g} / \mathrm{cm}^{2}$ (whereby $z=17.4 \mathrm{~cm}$ ) in the direction of the primary beam and an area of $30 \times 30 \mathrm{~cm}^{2}$ facing the beam. Such a thickness is typical of the storm shelters used by the crew during intense Solar Particle Events (SPEs) [4,14].

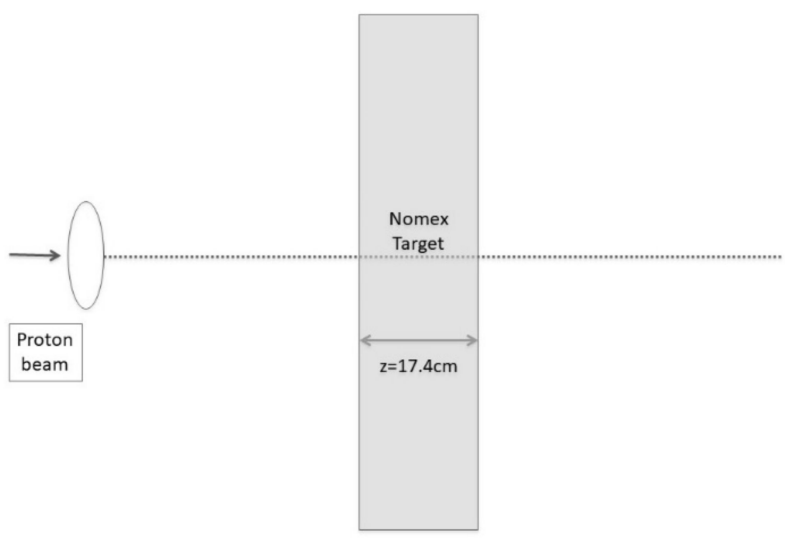

Figure 3. Experimental setup used in the simulation.

For each energy value, a run of $1.5 \times 10^{6}$ events was simulated. The Nomex chemical composition is shown in Table 1. 
Table 1. Thickness, density, and composition of the Nomex target used as a shield in space.

\begin{tabular}{cccc}
\hline Target & Thickness $\mathbf{( c m )}$ & $\boldsymbol{\rho}\left(\mathbf{g} / \mathbf{c m}^{3}\right)$ & Composition \\
\hline Nomex & 17.4 & 1.15 & {$[\mathrm{H}(4 \%) \mathrm{C}(54 \%) \mathrm{N}(9 \%) \mathrm{O}(10 \%) \mathrm{Cl}(23 \%)] 45 \%+[\mathrm{N}(70 \%) \mathrm{O}(30 \%)] 55 \%$} \\
\hline
\end{tabular}

The Table 2 shows the models implemented in Geant 4 and used DOSE. The energy cut thresholds are set to the Geant 4 default values.

Table 2. The models implemented in the Geant4 package and used in the DOSE application. Default parameters are used.

\begin{tabular}{cc}
\hline Particles & Models \\
\hline$e^{-}, e^{+}, \gamma$ & Penelope \\
\hline Protons & CHIPSElastic, Bertini, Binary \\
\hline Neutrons & HP Elastic, LElastic, CHIPSElastic, HP Inelastic Model, Bertini, Binary \\
\hline Ions & Binary \\
\hline
\end{tabular}

As already discussed, the shield that can be used in space must possess fundamental characteristics, including the capacity to absorb the secondary radiation generated by the interaction of the primary beam with the atomic nuclei of the chemical elements, this secondary radiation being the largest contribution to the dose absorbed by the astronauts. The choice of the Nomex [28,29] materials for this test is inspired by a previous study [15] concerning the shielding effectiveness of some materials suitable for applications in space. Nomex was the best in terms of dose reduction to astronauts in the case of the interaction with $1 \mathrm{GeV}$ protons [15]. In Nomex, there is a percentage of air due to the honeycomb structure. It is known that hydrogen is effective in shielding against GCR [30] because it breaks up the heavy ions by forming smaller, less harmful fragments [31], which are mostly absorbed inside the shield.

\section{Results: Mass, Charge, and Energy Distributions of Secondary Particles}

Figure 4 shows the yield of secondary particles as a function of the atomic and mass numbers ( $\mathrm{Z}$ and $\mathrm{A}$, respectively) produced in the proton-Nomex interaction. Since the production rates depend on the processes that open with the increase of proton energy, we investigate how the production of secondary particles evolves with the bombarding energy. We selected a few energy values within the energy spectrum of GCRs shown in Figure 1 to give an overview of such evolution.

At all energies, in the calculation results, we can identify three main production clusters located (1) at the larger masses around the mass of ${ }^{37} \mathrm{Cl}$, (2) around the masses of ${ }^{12} \mathrm{C}$ and ${ }^{14} \mathrm{~N}$, (3) in correspondence of the light-particle masses with atomic number $\mathrm{Z} \leq 2$. The presence of these three clusters provides the first indication that protons impinging on $\mathrm{C}, \mathrm{N}$, and $\mathrm{Cl}$ nuclei produce composite systems, as a consequence of the proton capture that deexcite by emitting light particles in a cascade. Particle emission can be evaporation-like (producing a Maxwellian energy spectrum, more likely at the lowest proton energy) or of the pre-equilibrium type (with a non-Maxwellian energy spectrum extended up to almost the beam energy). The mass range or width of clusters 2 and 3 depend on the excitation energy and increase with the proton energy. Such a trend is expected since the highest the excitation energy, the more particles are evaporated, and the lightest is the residual nucleus. As a consequence, on the light particle side (cluster 3), the increase of the excitation energy opens more decay channels, decay cascades are longer, and deuterons, tritons, and ${ }^{3,4} \mathrm{He}$ emissions become more probable. Such an increase is clearly visible in Figure 4 . The relative population of the ions that differ by one unit of mass and charge from ${ }^{12} \mathrm{C},{ }^{14} \mathrm{~N}$, and ${ }^{37} \mathrm{Cl}$ can also slightly change because direct reaction channels are opened at larger proton energy. However, this effect is limited to such mass and charge regions since protons can only produce knockout of protons or neutrons or 
a pickup of a neutron. Thus, the production of secondary protons and neutrons can also have a slight contribution from these direct channels. The extension of clusters 2 and 3 towards smaller masses and charges reaches saturation at energies above $200 \mathrm{MeV}$ because the wavelength of the relative motion becomes so small that nuclei are transparent to protons and productions of different particles (e.g., muons, pions, etc.) become the predominant channels via inelastic direct channels such as p-p and p-n. This behavior is also reflected in the spectra of the atomic number of the secondary fragment in Figure 5 and in the energy spectra shown in Figure 6. The energy spectra are dominated at the lowest energies by light particles, as, for instance, the emission from compound nuclei, while direct reactions are the dominant production processes at all beam energies. The energy spectra are consequently of continuous type. The energy peak at around the beam energy shows up at the highest energies being the consequence of the smaller wavelength and higher transparency of the nucleus to the incoming protons and the opening of the p-p and p-n direct inelastic interactions.
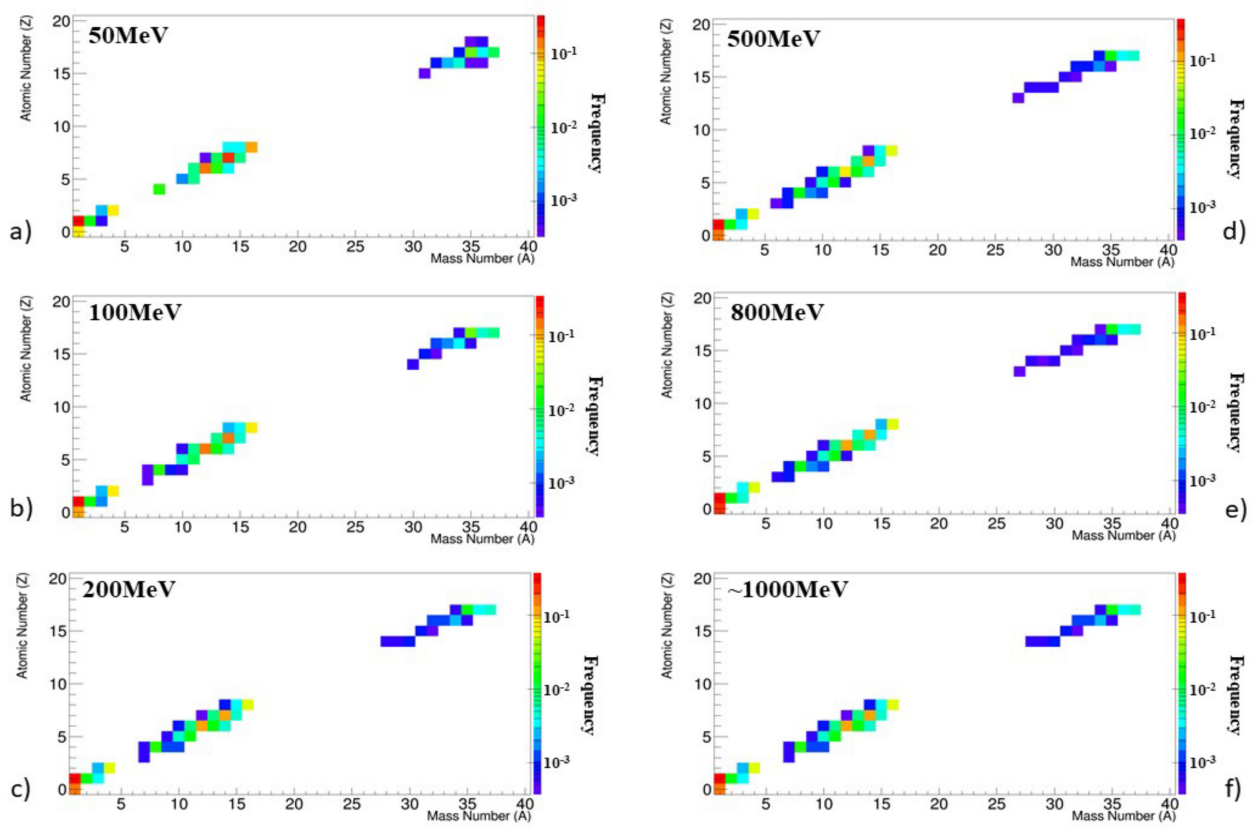

Figure 4. Computed atomic and mass numbers $(\mathrm{Z}, \mathrm{A})$ of the secondary particles produced as a result of the proton $\neg$-Nomex interaction for different energy values that characterize the spectrum of GCRs.

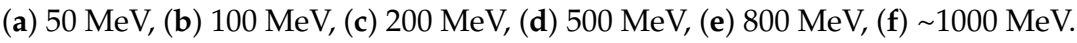

A further feature common at all energies is the copious production of neutrons. This is because merely all nuclear collisions (except elastic) that are involved produce neutrons. Being neutrons more penetrating than charged radiations, their contribution to the dose absorbed by the astronauts can be relatively very large. Therefore, the capability of the shielding material to reduce the escape of neutrons is a challenge for the development of any shielding material.

The overall picture coming from the mass, charge, and energy distributions of the secondary products is therefore consistent with expectations of the general trend of nuclear reaction mechanisms with the bombarding energy. It is important to remark that, as a second step, the code DOSE takes care of the transport of these secondary particles in the medium. In principle, such particles, besides being slowed down by electromagnetic interactions, may interact again with the nuclei in the medium and give rise to hadronic processes (capture, inelastic processes, evaporation) of the same type of the one already discussed. Therefore, the check on the reliability of the implementation of those processes that forge secondary particles gives confidence that the transport of secondary radiation is also performed in a reliable manner. 

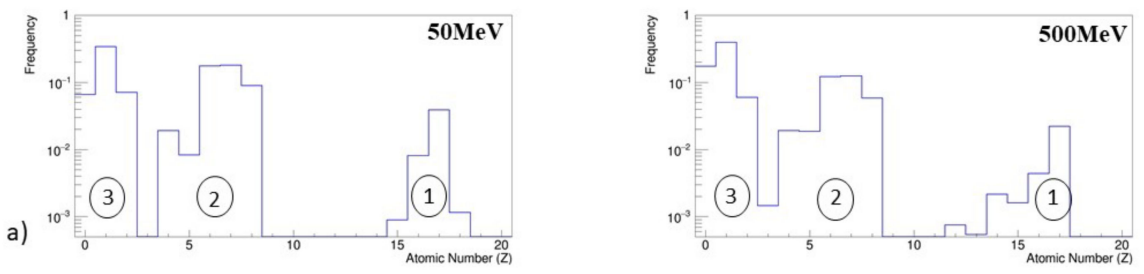

d)
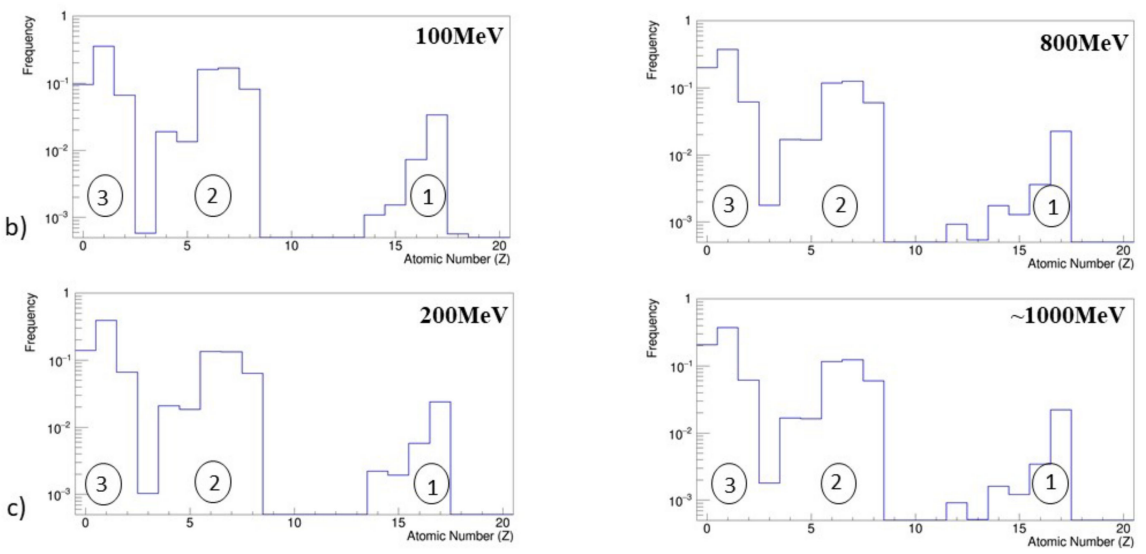

Figure 5. Computed atomic numbers and event frequency of the secondary particles produced as a result of the proton-Nomex interaction for different energy values that characterize the spectrum of GCRs. The three clusters of high (1), middle (2), and low (3) masses are highlighted. (a) $50 \mathrm{MeV}$, (b) $100 \mathrm{MeV}$, (c) $200 \mathrm{MeV}$, (d) $500 \mathrm{MeV}$, (e) $800 \mathrm{MeV}$, (f) $1000 \mathrm{MeV}$.
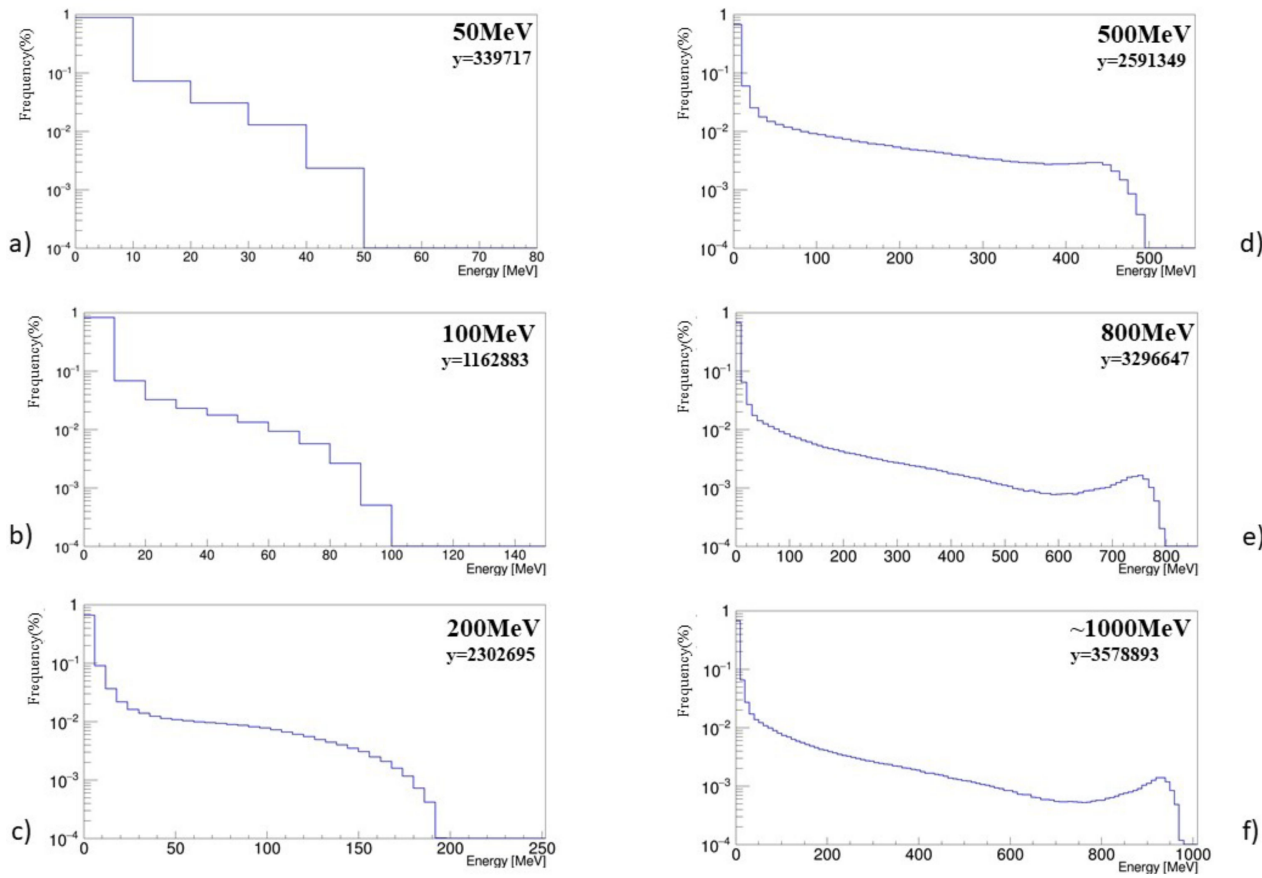

Figure 6. Energy spectra for the secondary products. For each energy, the spectrum is independently normalized to the total yield (y). (a) $50 \mathrm{MeV}$, (b) $100 \mathrm{MeV}$, (c) $200 \mathrm{MeV}$, (d) $500 \mathrm{MeV}$, (e) $800 \mathrm{MeV}$, (f) $\sim 1000 \mathrm{MeV}$.

\section{Summary and Conclusions}

In this work, we explored the production of secondary radiations produced in the interaction of a proton beam of energies up to $1 \mathrm{GeV}$ with a shield made with Nomex material of the known chemical composition. The code DOSE was used to simulate the interaction of the protons with the atoms in the shield. We have analyzed the production of 
secondary radiation in the target from the point of view of the implemented processes. We remarked the production of ions of masses and charges lower than the original chemical elements, but also a consistent production of neutrons, protons, and alpha particles.

As secondary radiation can escape the shield and possibly be more harmful than the incoming radiation (for instance, neutrons because of their higher penetrability), it is necessary to consider the transport of this secondary radiation in the same shielding material and to evaluate the dose absorbed as a function of the distance from the shield. This part of the work will be the subject of an article in preparation.

Author Contributions: Conceptualization, F.L., E.V. and M.Q.; methodology, F.L., E.V. and M.Q.; software, F.L. and D.B.; validation, F.L., E.V. and M.Q.; formal analysis, F.L., D.B., A.D.N.; investigation, F.L, and E.V.; resources, F.L. and M.Q.; data curation, F.L., E.V. and M.Q.; writing-original draft preparation, F.L., E.V. and M.Q.; writing—review and editing, F.L., E.V., D.B., A.D.N. and M.Q.; visualization, F.L., E.V., D.B., A.D.N. and M.Q.; supervision, F.L and E.V.; project administration, F.L., E.V. and M.Q.; funding acquisition, F.L. and M.Q. All authors have read and agreed to the published version of the manuscript.

Funding: This research has been supported by the University of Naples Federico II, the Italian Ministry of University and Research (MIUR), within the program PON R\&I 2014-2020- Attraction and International Mobility (AIM)- CUP E61G19000020001.

Institutional Review Board Statement: Not applicable.

Informed Consent Statement: Not applicable.

Data Availability Statement: Supporting data includes data generated at the MC simulation. All of the data supporting the findings of the presented study are available from the corresponding author on request.

Conflicts of Interest: The authors declare no conflict of interest.

\section{References}

1. Barcellos-Hoff, M.H.; Blakely, E.A.; Burma, S.; Fornace, A.J., Jr.; Gerson, S.; Hlatky, L.; Kirsch, D.G.; Luderer, U.; Shay, J.; Wang, Y.; et al. Concepts and challenges in cancer risk prediction for the space radiation environment. Life Sci. Space Res. 2015, 6, 92-103. [CrossRef]

2. Shea, M.; Smart, D. Space weather: The effects on operations in space. Adv. Space Res. 1998, 22, 29-38. [CrossRef]

3. Hellweg, C.E.; Baumstark-Khan, C. Getting ready for the manned mission to Mars: The astronauts' risk from space radiation. Naturwissenschaften 2007, 94, 517-526. [CrossRef] [PubMed]

4. Durante, M.; Cucinotta, F.A. Physical basis of radiation protection in space travel. Rev. Mod. Phys. 2011, 83, 1245. [CrossRef]

5. Thomas, S.R.; Owens, M.J.; Lockwood, M. Galactic cosmic rays in the heliosphere. Astron. Geophys. 2014, 55, 23-25. [CrossRef]

6. Chowdhury, P.; Kudela, K.; Moon, Y.J. A Study of Heliospheric Modulation and Periodicities of Galactic Cosmic Rays during Cycle 24. Sol. Phys. 2016, 291, 581-602. [CrossRef]

7. Guo, J.; Zeitlin, C.; Wimmer-Schweingruber, R.F.; Hassler, D.M.; Ehresmann, B.; Rafkin, S.; Freiherr von Forstner, J.L.; Khaksarighiri, S.; Liu, W.; Wang, Y. Radiation environment for future human exploration on the surface of Mars: The current understanding based on MSL/RAD dose measurements. Astron. Astrophys. Rev. 2021, 29, 8. [CrossRef]

8. Schimmerling, W.; Cucinotta, F.; Wilson, J. Radiation risk and human space exploration. Adv. Space Res. 2003, 31, 27-34. [CrossRef]

9. Shen, Z.-N.; Quin, G. Modulation of Galactic Cosmic Rays in the Inner Heliosphere over Solar Cycles. Astrophys. J. 2018, 854, 137. [CrossRef]

10. SPace Environment Information System SPENVIS. Available online: https:/ / www.spenvis.oma.be/ (accessed on 20 January 2022).

11. Tylka, A.J.; Adams, J.H.; Boberg, P.R.; Brownstein, B.; Dietrich, W.F.; Flueckiger, E.O.; Petersen, E.L.; Shea, M.A.; Smart, D.F.; Smith, E.C. CREME96: A Revision of the Cosmic Ray Effects on Micro-Electronics Code. IEEE Trans. Nucl. Sci. 1997, 44, 2150-2160. [CrossRef]

12. Nymmik, R.A.; Panasyuk, M.I.; Pervaja, T.I.; Suslov, A.A. A Model of Galactic Cosmic Ray Fluxes. Nucl. Tracks Radiat. Meas. 1992, 20, 427-429. [CrossRef]

13. Nymmik, R.A.; Panasyuk, M.I.; Suslov, A.A. Galactic cosmic ray flux simulation and prediction. Adv. Space Res. 1996, 17, 2. [CrossRef]

14. Loffredo, F.; Vardaci, E.; Quarto, M.; Roca, V.; Pugliese, M. Validation of electromagnetic and hadronic physical processes in the interaction of a proton beam with matter: A Solar Particle Events case study with an Al slab. Adv. Space Res. 2017, 59, 393. [CrossRef]

15. Loffredo, F.; Vardaci, E.; Roca, V.; Pugliese, M. Space missions: Comparison of shielding effectiveness among different materials using $1 \mathrm{GeV}$ protons. Mater. Res. Express 2019, 6, 016544. [CrossRef] 
16. Lechner, A. Particle interactions with matter. CERN Yellow Rep. School Proc. 2018, 5, 47.

17. Cucinotta, F.A.; Katz, R.; Wilson, J.W.; Townsend, L.W.; Shinn, J.; Hajnal, F. Biological Effectiveness of High-Energy Protons: Target Fragmentation. Radiat. Res. 1991, 127, 130-137. [CrossRef] [PubMed]

18. Cucinotta, F.A.; Atwell, W.; Weyland, M.; Hardy, A.C.; Wilson, J.W.; Townsend, L.W.; Shinn, J.L.; Katz, R. Radiation Risk Predictions for Space Station Freedom Orbits; NASA TP 3098; National Aeronautics and Space Administration: Washington, DC, USA, 1991.

19. Zeitlin, C. Space Radiation Shielding. In Handbook of Bioastronautics; Young, L.R., Sutton, J.P., Eds.; Springer: Cham, Switzerland, 2021; pp. 353-375. [CrossRef]

20. Norbury, J.W.; Slaba, T.C.; Aghara, S.; Badavi, F.F.; Blattnig, S.R.; Clowdsley, M.S.; Heilbronn, L.H.; Lee, K.; Maung, K.M.; Mertens, C.J.; et al. Advances in space radiation physics and transport at NASA. Life Sci. Space Res. 2019, 22, 98-124. [CrossRef]

21. Ivantchenko, A.V.; Ivanchenko, V.N.; Quesada Molina, J.; Incerti, S.L. Geant4 hadronic physics for space radiation environment. Int. J. Radiat. Biol. 2012, 88, 171-175. [CrossRef]

22. Allison, J.; Amako, K.; Apostolakis, J.; Araujo, H.; Arce Dubois, P.; Asai, M.; Barrand, G.; Capra, R.; Chauvie, S.; Chytracek, R.; et al. Geant4 Developments and Applications. IEEE Trans. Nucl. Sci. 2006, 53, 270-278. [CrossRef]

23. Agostinelli, S. The Geant4 Collaboration. GEANT4-A simulation toolkit. Nucl. Instrum. Methods Phys. Res. A 2003, 506, 250-303. [CrossRef]

24. Mancusi, D.; Bertucci, A.; Gialanella, G.; Grossi, G.; Manti, L.; Pugliese, M.; Rusek, A.; Scampoli, P.; Sihver, L.; Durante, M. Comparison of aluminum and lucite for shielding against $1 \mathrm{GeV}$ protons. Adv. Space Res. 2007, 40, 581-585. [CrossRef]

25. Bertucci, A.; Durante, M.; Gialanella, G.; Grossi, G.; Manti, L.; Pugliese, M.; Scampoli, P.; Mancusi, D.; Sihver, L.; Rusek, A. Shielding of relativistic protons. Radiat. Environ. Biophys. 2007, 46, 107-111. [CrossRef] [PubMed]

26. Lobascio, C.; Briccarello, M.; Destefanis, R.; Faraud, M.; Gialanella, G.; Grossi, G.; Guarnieri, V.; Manti, L.; Pugliese, M.; Rusek, A.; et al. Accelerator-based tests of radiation shielding properties of materials used in human space infrastructures. Health Phys. 2008, 94, 242-247. [CrossRef] [PubMed]

27. Berger, M.J.; Coursey, J.S.; Zucker, M.A.; Chang, J. ESTAR, PSTAR, and ASTAR: Computer Programs for Calculating Stopping-Power and Range Tables for Electrons, Protons, and Helium Ions (Version 1.2.3); National Institute of Standards and Technology: Gaithersburg, MD, USA, 2005.

28. Loffredo, F.; Vardaci, E.; Roca, V.; Pugliese, M. Nomex with boron as a neutron shielding in space: Preliminary study. Il Nuovo Cimento C 2018, 41, 220.

29. Loffredo, F.; Vardaci, E.; Pugliese, M.; Serra, M.; Quarto, M. Dosimetry in Space: The shielding effectiveness for the radioprotection of astronauts against $1 \mathrm{GeV}$ protons. Il Nuovo Cimento C 2020, 43, 133.

30. Wilson, J.W.; Cucinotta, F.A.; Miller, J.; Shinn, J.L.; Thibeault, S.A.; Singleterry, R.C.; Simonsen, L.C.; Kim, M.H. Materials for Shielding Astronauts from the Hazards of Space Radiations. Mat. Res. Soc. Symp. Proc. 1999, 551, 3-15. [CrossRef]

31. Bondorf, J.P.; Botvina, A.S.; Iljinov, A.S.; Mishustin, I.N.; Sneppen, K. Statistical multifragmentation of nuclei. Phys. Rep. 1995, 257, 133-221. [CrossRef] 